\title{
Large Eddy Simulation of the Flow Past a Soccer Ball
}

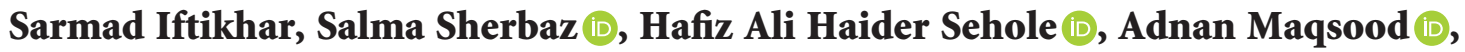 \\ and Zartasha Mustansar $(\mathbb{D}$
}

Research Centre for Modeling \& Simulation (RCMS), National University of Sciences and Technology (NUST), H-12, Islamabad 4400, Pakistan

Correspondence should be addressed to Adnan Maqsood; adnan@rcms.nust.edu.pk

Received 31 July 2021; Revised 11 December 2021; Accepted 27 December 2021; Published 18 January 2022

Academic Editor: Hao Zhang

Copyright (๑) 2022 Sarmad Iftikhar et al. This is an open access article distributed under the Creative Commons Attribution License, which permits unrestricted use, distribution, and reproduction in any medium, provided the original work is properly cited.

\begin{abstract}
The football game is the most popular, played, and loved sport around the world. The advent of technological breakthroughs and the continuous increase in consumer demand have led to a revolution in football's design and manufacturing process. In the past, studies in soccer ball aerodynamics mainly were limited to the investigation of lift and drag forces inside a wind tunnel apparatus. A few researchers have analyzed the flow around the different soccer balls using computational fluid dynamics simulations with the Reynolds-Averaged-Navier-Stokes equations model. This study primarily intends to simulate a modern soccer ball (Adidas Telstar 18) using the Large Eddy Simulations technique. The whole research is divided into two phases. In the first phase, the flow around a smooth sphere is simulated numerically to validate the meshing strategy, boundary conditions, and solution methodology. The same modeling approach is used in the later stage to simulate the flow around a soccer ball. The effect of panels and seam on the boundary layer flow separation and overall turbulent flow structure around the soccer ball are visualized. The results indicate that the large-eddy simulations help predict the flow intricacies by resolving small eddies near the panels.
\end{abstract}

\section{Introduction}

There has been a significant increase in the research studies utilizing Computational Fluid Dynamics (CFD) techniques in engineering design, optimization, structure analysis, and many other applications [1]. Several general-purpose CFD codes, such as OpenFoam, Fluent, CFX, X-FLOW, COMSOL, STAR-CCM+, etc., are used to perform such studies. For the past two decades, CFD has had a massive influence on sports stadia and equipment design [2-4]. It has played an essential role in understanding and improving the performance of various sports projectiles [5].

The previous research conducted in the area of soccer ball aerodynamics can be bifurcated into two main categories. The first category mainly covers the aerodynamic performance assessment of different soccer balls using wind tunnel testing and CFD methods. The other type deals with the research related to the soccer ball trajectory analysis. A brief overview of the latest experimental and numerical research studies dealing with the soccer ball performance assessment is given in the subsequent paragraphs.
Carré et al. [6] used wind tunnel measurements to study how the transition of the boundary layers from laminar to turbulent altered the drag coefficient of a soccer ball at a high Reynolds number. The reverse Magnus effects were noticed for spinning balls at low Reynolds numbers. Asai et al. [7, 8] performed wind tunnel experimentation to compare the aerodynamic coefficients of the soccer balls under static and rotating conditions. The vortex dynamics during the balls' flight were analyzed using the titanium tetrachloride visualization method. Visualization experiments for a nonrotating ball revealed that the boundary-layer separation point is approximately $90^{\circ}$ at a slow-kick-induced at a speed of $5 \mathrm{~m} / \mathrm{s}$ - and approximately $120^{\circ}$ during a fast kick-induced at a speed of $29 \mathrm{~m} / \mathrm{s}$. The experimental study conducted by Oggiano and Sætran [9] focused on measuring different soccer balls' drag and side forces in static and spinning conditions. Free kick simulations were also performed by implementing the experimental data in a Matlab ${ }^{\circledR}$ routine. It was concluded that the panel shapes, panel numbers, surface dimples, and different seams carry substantial implications for the flight trajectories of the other soccer balls. Passmore 
et al. [10] conducted wind tunnel testing to measure important factors, such as Reynolds number sensitivity, Magnus effects, low spin rate orientation (knuckle), and unsteady aerodynamic loads of several FIFA ${ }^{\circledR}$-approved footballs. It was concluded that the different drag characteristics and unsteady aerodynamic loads of these soccer balls had only slightly impacted their flight performance. At the same time, a considerable change of lateral forces produced a significant difference in the flight trajectories of soccer balls with slight rotation and spin.

The purpose of the experimental study conducted by Asai et al. [11] was to compare the fundamental aerodynamic attributes of two different football specimens. The first specimen was the Adidas Tango 12, having 8 panels, while the second specimen was similar to the one used in Euro 2012, which had 32 panels. A higher critical Reynolds number $\left(3.3 \times 10^{5}\right)$ was observed for 8-panel Adidas compared to 32panel Euro $2012\left(2.4 \times 10^{5}\right)$. Similarly, the drag coefficient of the Euro 2012 ball was more comparable to a golf ball than the alternate Adidas Tango 12. Asai and Seo [12] performed a steady-state analysis of the four different soccer balls with varied panels, i.e., Adidas Tango 12, Adidas Roteiro, Adidas Teamgeist II, and Adidas Jabulani, each manufactured with $32,32,14$, and 8 panels, respectively. The parameters recorded during these experiments include drag coefficient and critical Reynolds numbers. The drag coefficient's impact on the flight range and trajectories was analyzed with the help of a simple 2D flight trajectory simulation. It revealed that the Reynolds number and the total seam length had a strong correlation. Alam et al. [13-15] experimentally evaluated with aerodynamic forces and moments for several FIFA-approved soccer balls under different wind conditions. Another experimentation of similar nature was undertaken by Alam et al. [16]. The study involved the investigation of aerodynamics attributes of 6-soccer balls, each having a variable surface structure. The results revealed that the drag coefficients and nature of fluid flow on the surface of soccer balls drew significant bearing from their surface structure. Hong et al. [17] experimentally investigated the flight pattern and aerodynamic attributes of four soccer balls, each with a particular panel shape and panel number. The experimental results indicated that the panel number and their relative orientation played a significant role in determining the aerodynamic performance of soccer balls.

Similarly, the panel shape and orientation were more important as far as the flight trajectory of the soccer balls was concerned. Goff et al. [18] reported wind tunnel measurement of the drag coefficient for nonspinning Jabulani and Brazuca balls. In the case of Brazuca, critical drag speed was found lower than that of Jabulani, whereas the supercritical drag coefficient was much high. The recorded drag data was also used in simulating the trajectories of these soccer balls. Hong et al. [19] experimentally studied the effect of surface dimples vis-a-vis aerodynamic attributes of the ten different soccer balls. They confirmed that dimples had a considerable impact on the aerodynamic forces acting on their surface. A similar experimental study performed more recently by Hong and Asai [20] tested the aerodynamics of the five different 32-panel soccer balls in the wind-tunnel assembly.
Because of high computational power requirements and possibly a previous lack of demand for a highly detailed understanding, relatively few research studies have attempted to simulate the flow around a different soccer ball. Carré et al. [1] also analyzed the effect of the incoming flow velocity and spin conditions on the aerodynamic properties of a typical soccer ball using wind tunnel testing and CFD. Barber et al. [21] performed a CFD assessment of the ball's aerodynamic behavior based on seam width and sharpness. In another numerical study conducted by Barber et al. [22], Reynolds Averaged Navier-Stokes equations with realizable $\mathrm{k}-\varepsilon$ turbulence model were used to calculate the drag, lift, side force coefficients, and pressure distributions close to the stagnation point of a soccer ball. Jalilian et al. [23] used CFD to analyze the effects of spin rate and surface pattern on aerodynamic forces acting on different sports balls (baseball, volleyball, and two soccer balls). It was noticed that the spin rate and the drag and lift forces acting on these balls were closely associated. Rohr [24] estimated the aerodynamic significance of seam while applying the CFD technique on a nonrotating twodimensional demonstration of the soccer ball. This study used a transitional solver to model the influence of seam on the boundary layer and overall transient flow structure. The author reported a local effect of the seam on the skin friction. However, this effect was not strong enough to delay the separation point as predicted by previous literature. Hussain et al. [25] performed a numerical study of a smooth sphere and a 32-panel conventional soccer ball to analyze the correlation between the shape of the ball and the corresponding aerodynamic attributes. K-epsilon turbulence model was used. The calculated results were examined because of the provided numerical and experimental findings. Asai et al. [26] used the lattice Boltzmann method, wind tunnel testing, and free-flight experiments to investigate the aerodynamic characteristics and flow field around a soccer ball. It was concluded that the trajectory of the spinning ball is stable and regular even after the deflection because of the shifting of the boundary layer separation points.

During the literature review, it was observed that most of the early studies in the area of soccer ball aerodynamics relied on wind tunnel experiments, typically collecting force data. A very few research studies have performed flow visualization using a particle image velocimetry measurement. A relatively few research studies have attempted to simulate the flow around different soccer balls because of high computational power requirements and possibly a previous lack of demand for a more comprehensive understanding. Hence, a detailed CFD analysis for practical comparative estimation of the complex turbulent flow field around a soccer ball is required to understand the effect of ball seam and panel designs on flow physics. The instant study aims to bridge the gaps in computational studies conducted in the past by modeling the flow around a modern soccer ball using high-fidelity large-eddy simulation techniques. The complete study is divided into two phases. In the first phase, the turbulent airflow around a static smooth sphere is investigated numerically for a range of Reynolds numbers using large-eddy simulations. In the later stage, the same turbulence modeling approach simulates the flow around a soccer ball. 


\section{Governing Equations}

In the present research, the three-dimensional flow of a viscous, incompressible fluid is considered. The resulting governing equation, i.e., continuity and momentum, take shape [27].

$$
\begin{gathered}
\frac{\partial u_{i}}{\partial x_{i}}=0 \\
\frac{\partial u_{i}}{\partial t}+u_{j} \frac{\partial u_{i}}{\partial x_{j}}=-\frac{1}{\rho} \frac{\partial p}{\partial x_{i}}+\nu \frac{\partial^{2} u_{i}}{\partial x_{j} \partial x_{i}},
\end{gathered}
$$

where $u_{i}$ denotes the velocity components in Cartesian coordinates, $p$ represents the pressure, $\rho$ stands for the density, and $v$ is the kinematic viscosity. The initial set of equations and the energy equation are mostly called Naiver Stokes (NS) equations. A turbulence model is required to numerically solve the governing unsteady NS equations for any turbulent flow problem.

Solving the above equations without making further simplifying assumptions is called direct numerical simulation (DNS). This method does not mandate more closure correlations because it directly solves the unsteady NS equations. Since the DNS method can resolve the minuscule eddies formation and time scales of turbulence, extremely small time steps and very fine grids are required to find accurate solutions. Because of these restrictions, the DNS method is mainly applied to low Reynolds number flow applications with small-size computation domains. Therefore, DNS possesses limited applicability to simulate the current high Reynolds number complex flow problem $[28,29]$.

Another option is the use of large-eddy simulation (LES) solvers, which accurately predict the large-scale turbulent structures and use the subgrid-scale model to represent the smaller-scale eddies within the flow domain. It results in a significant reduction in the computational effort as compared to direct numerical simulation. Though LES only resolves the large-scale eddies, an extremely fine grid is still required. The practicality of this method to resolve complex flow problems associated with high Reynolds numbers has stood the test of time $[30,31]$. In the present study, the governing equations employed for LES are obtained by filtering the time-dependent Navier-Stokes equations. The resulting equations are as follows [30]:

$$
\begin{gathered}
\frac{\partial \bar{u}_{i}}{\partial x_{i}}=0 \\
\frac{\partial \bar{u}_{i}}{\partial t}+\frac{\partial \bar{u}_{i} \bar{u}_{j}}{\partial x_{j}}=-\frac{1}{\rho} \frac{\partial \bar{p}}{\partial x_{i}}+v \frac{\partial^{2} \bar{u}_{i}}{\partial x_{j} \partial x_{i}}-\frac{\partial \tau_{i j}}{\partial x_{j}},
\end{gathered}
$$

where $\bar{u}_{i}$ and $\bar{p}$ represent the grid-filtered values of the velocity and the pressure, respectively. $\tau_{i j}$ is the subgridscale stress and is defined as

$$
\tau_{i j}=\overline{u_{i} u_{j}}-\bar{u}_{i} \bar{u}_{j}
$$

The above subgrid-scale stresses resulting from the filtering operation are unknown and modeled with a subgridscale model.

$$
\tau_{i j}-\frac{1}{3} \tau_{k k} \delta_{i j}=-2 \mu_{t} \bar{S}_{i j}
$$

The term $\tau_{k k}$ is the isotropic part of the subgrid-scale stresses, which is added to the filtered static pressure term, whereas $\mu_{t}$ and $\bar{S}_{i j}$ are the subgrid-scale turbulent viscosity and rate-of-strain tensor for the resolved scale, respectively.

$$
\bar{S}_{i j}=\frac{1}{2}\left(\frac{\partial \bar{u}_{i}}{\partial x_{j}}+\frac{\partial \bar{u}_{j}}{\partial x_{i}}\right) .
$$

To model $\mu_{t}$, ANSYS ${ }^{\circledR}$ FLUENT offers four models, i.e., Smagorinsky-Lilly model, the dynamic Smagorinsky-Lilly model, wall-adapting local eddy viscosity model (WALE model), and the dynamic kinetic energy subgrid-scale model. In the WALE model, the eddy viscosity is modeled by

$$
\mu_{t}=\rho L_{s}^{2} \frac{\left(S_{i j}^{d} S_{i j}^{d}\right)^{3 / 2}}{\left(\bar{S}_{i j}^{d} \bar{S}_{i j}^{d}\right)^{5 / 2}+\left(S_{i j}^{d} S_{i j}^{d}\right)^{5 / 4}},
$$

where $L_{s}$ and $S_{i j}^{d}$ are defined as

$$
\begin{aligned}
L_{s} & =\min \left(\kappa d, C_{w} V^{1 / 3}\right), \\
S_{i j}^{d} & =\frac{1}{2}\left(\bar{g}_{i j}^{2}+\bar{g}_{j i}^{2}\right)-\frac{1}{3} \delta_{i j} \bar{g}_{k k}^{2}, \\
\bar{g}_{i j} & =\frac{\partial \bar{u}_{i}}{\partial x_{j}} .
\end{aligned}
$$

The value of the WALE constant $C_{w}$ used in the current case is 0.325 .

\section{CFD Framework}

The development of a computational setup for flow analysis will be discussed in the subsequent paragraphs.

3.1. Geometry. Modern soccer balls (Adidas Telstar18) with six thermally bonded panels and a diameter of $220 \mathrm{~mm}$ and a smooth sphere having the same diameter as the soccer ball are used in the current study. Adidas Telstar18 was used in FIFA world cup 2018. The 3D model of the soccer ball and smooth sphere was created in Ansys ${ }^{\circledR}$ (shown in Figure 1). The panel shape is highlighted in yellow color in Figure 1. The width and depth of the panel joints (seams) on the soccer ball surface are around $3.15 \mathrm{~mm}$ and $1.09 \mathrm{~mm}$, respectively.

3.2. Computational Domain and Grid Generation. The computational domain is rectangular, where the inlet and outlet peripheries are situated at a separation of $5 D$ and $10 D$, respectively ( $D$ is the diameter of the sphere/soccer ball). Similarly, the bottom and sides of the domain are located at a distance of $5 D$ from the soccer ball/sphere surface. 


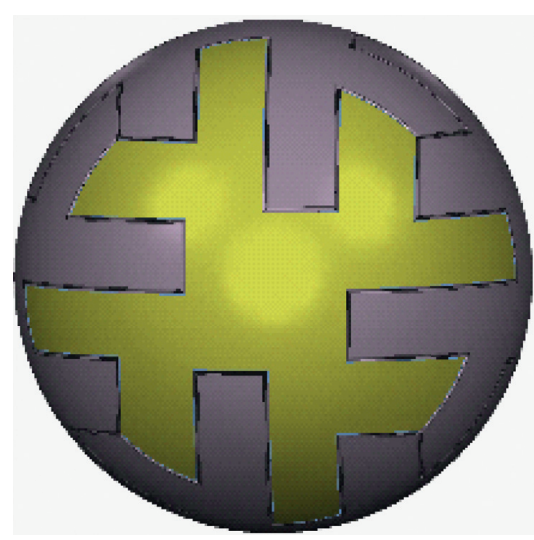

(a)

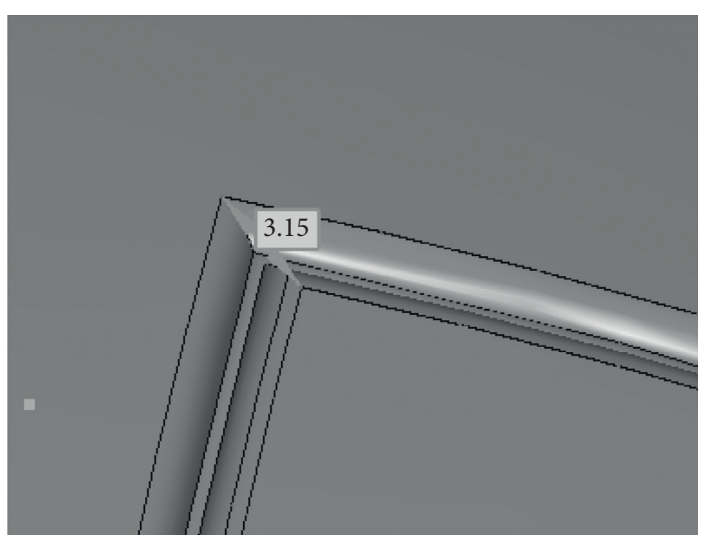

(b)

Figure 1: (a) 3D models of Telstar18 and (b) seam configuration on the surface of the soccer ball.

The process of dividing the domain of interest into several small portions/chunks is termed grid/mesh generation. High quality, appropriate grid generation is considered one of the critical factors to obtain accurate CFD results. Simulation time, stability, and the convergence of the solution are also affected by the quality and type of the mesh used. Mesh generation is usually considered to be the most time-taking process in CFD analysis. The triangular mesh can accurately capture any shape of interest and is generally used when the geometry is very complex.

In the present case, a triangular mesh is generated using ANSYS ${ }^{\circledR}$. In LES, mesh resolution governs the fraction of energy spectrum directly resolved. Our goal is to have a mesh fine enough to resolve $80 \%$ of the turbulent kinetic energy in the present study. To capture the boundary layer separation more accurately, ten prism layers were created along the normal-wall direction starting from the ball's surface (shown in Figure 2). The first cell in the prism layer is taken as $0.07 \mathrm{~mm}$, corresponding to a $y^{+}$value approximately equal to 1 . Figures $3(\mathrm{a})$ and $3(\mathrm{~b})$ show the distribution of $y^{+}$ on the surface of the sphere and soccer ball, respectively, calculated at an incoming flow velocity of $35 \mathrm{~m} / \mathrm{s}$. It can be observed that the calculated average value of $y^{+}$is less than one on the whole boundary surface in both cases.

3.3. Boundary Conditions and Solver Settings. The boundary conditions, namely velocity inlet at inlet boundary and pressure outlet at the outlet boundary, are prescribed. A nonslip wall condition is imposed at the soccer ball surface and a free slip condition at all sidewall boundaries.

Three-dimensional, incompressible transient flow simulations are performed using ANSYS $^{\circledR}$ Fluent. The walladapting local eddy viscosity (WALE) model is used for subgrid-scale turbulence modeling. A pressure-based solver is used with implicit time integration. Moreover, the pressure velocity coupling is catered with the coupling algorithm. The bounded central differencing scheme is used as a convection discretization scheme for all transport equations. The scheme is usually considered an ideal choice for LES simulations owing to its meritoriously low numerical diffusion. [32, 33]. A bounded second-order scheme is utilized for temporal discretization as recommended in the literature [32]. Simulations are performed on a high-end computing cluster.

\section{Results and Discussion}

Firstly, the flow over a smooth sphere having the same dimension as that of a soccer ball $(0.22 \mathrm{~m})$ is simulated to validate the meshing strategy, boundary conditions, and solution methodologies. LES simulations are performed at several Reynolds numbers (Re) to cover the range of flow regimes duly represented by laminar steady-state flow ranging $\operatorname{Re}=100$ and turbulent flow up to $\operatorname{Re}=10^{6}$. The available experimental/numerical data at the same flow conditions are used for validation purposes [34, 35]. The comparison between the computed and available results of drag coefficients at different Reynolds numbers is shown in Figure 4. It can be observed that the calculated values agree well with experimental results. The pressure distribution around the smooth sphere in the three different flow regimes, i.e., subcritical and critical and supercritical flow regimes, are plotted in Figures 5(a)-5(c), respectively. The corresponding Reynolds number is $\mathrm{Re}=1 \times 10^{4}$, $\operatorname{Re}=3.18 \times 10^{5}$, and $\operatorname{Re}=1.41 \times 10^{6}$, respectively. The polar angle $\varphi$ is measured from the front stagnation point. The calculated results are compared with Achenbach's experimental [34] and M. Muto's numerical data values [35]. It can be concluded that the calculated values are in good agreement with the available experimental/numerical findings. Hence, the same turbulence modeling approach, meshing strategy, boundary conditions, and solution methodologies are used in the second phase of the study, i.e., simulating flow around the soccer ball.

The soccer ball is primarily played in the wind speed range of $7 \mathrm{~m} / \mathrm{s}$ to $35 \mathrm{~m} / \mathrm{s}$. It corresponds to the speed range of $16 \mathrm{mph}$ to $78 \mathrm{mph}$ and a Reynolds number range of $10^{5}<\operatorname{Re}<5 \times 10^{5}$ [36]. In the present study, the numerical simulations are performed at Reynold number of $5 \times 10^{5}$ $(35 \mathrm{~m} / \mathrm{s})$. A comparison of experimental and calculated values of drag coefficient is shown in Table 1. 


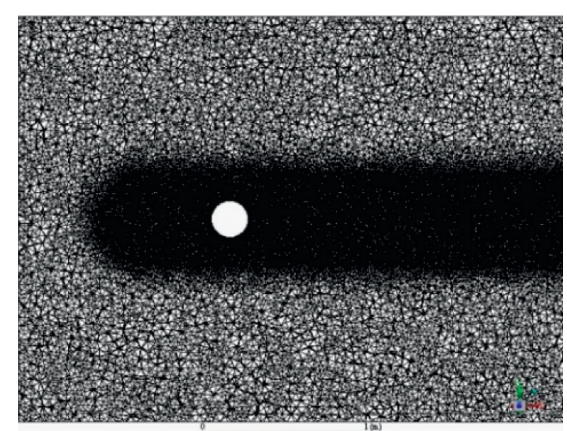

(a)

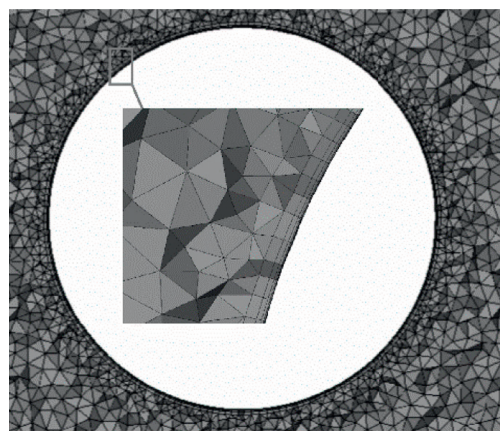

(b)

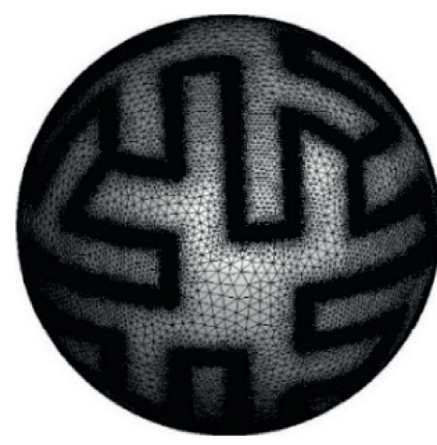

(c)

Figure 2: .(a) Mesh around sphere. (b) Zoom-up view of sphere-surface prism layer grids. (c) Mesh on the soccer ball surface.

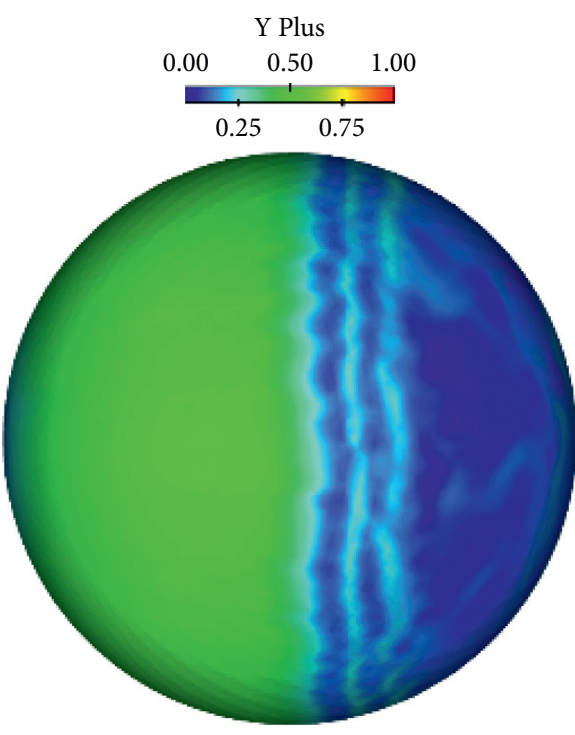

(a)

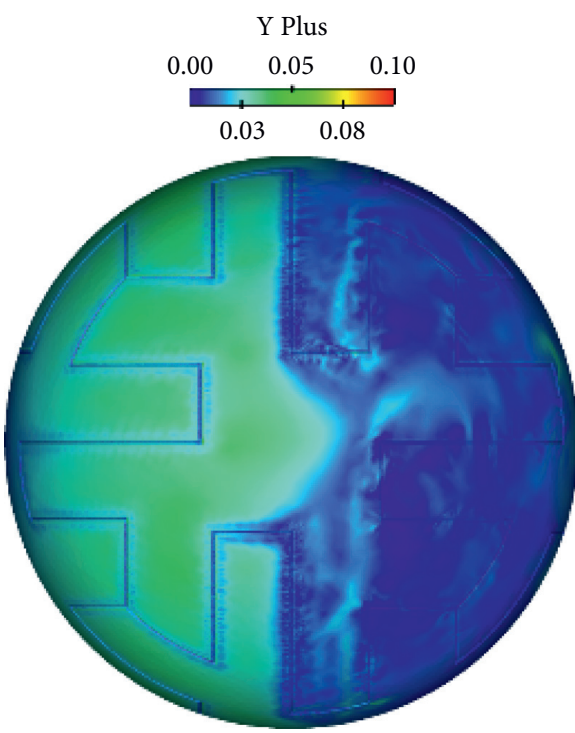

(b)

Figure 3: (a) Calculated $y^{+}$distribution on (a) sphere and (b) soccer ball.

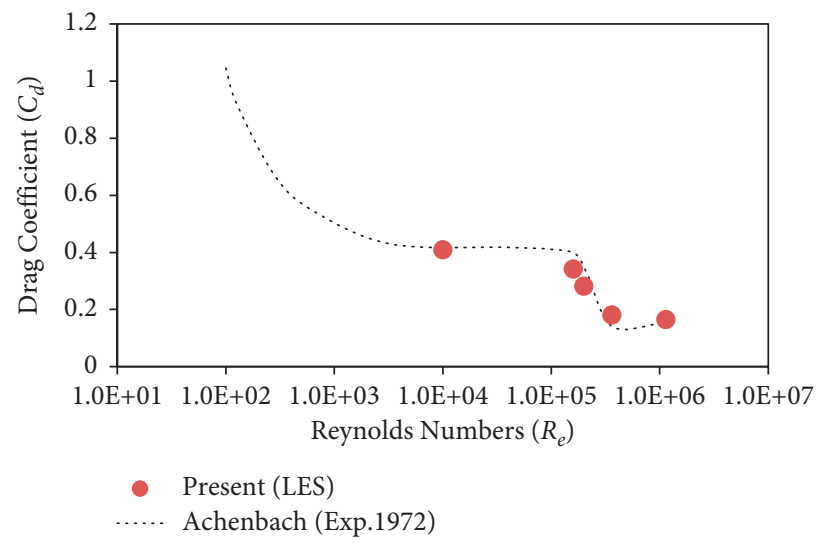

Figure 4: Comparison between the calculated values of drag coefficient with experimental results. 


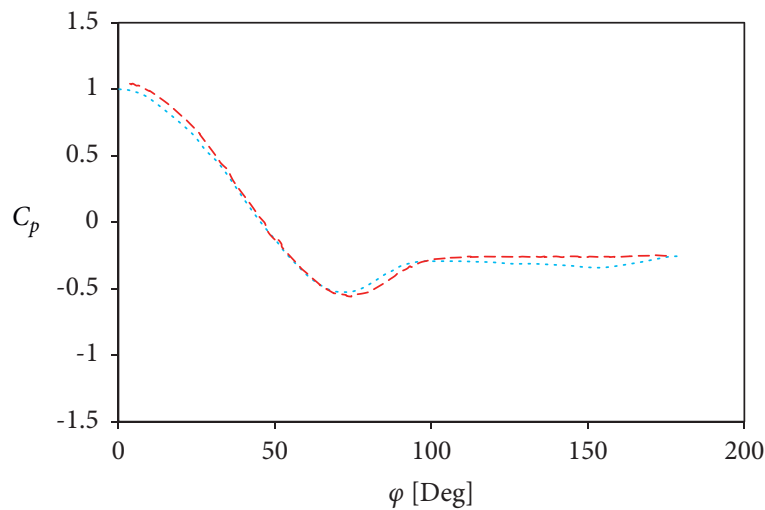

M. Muto 1.E4 (LES,2012)

- - - Present sphere 1.E4

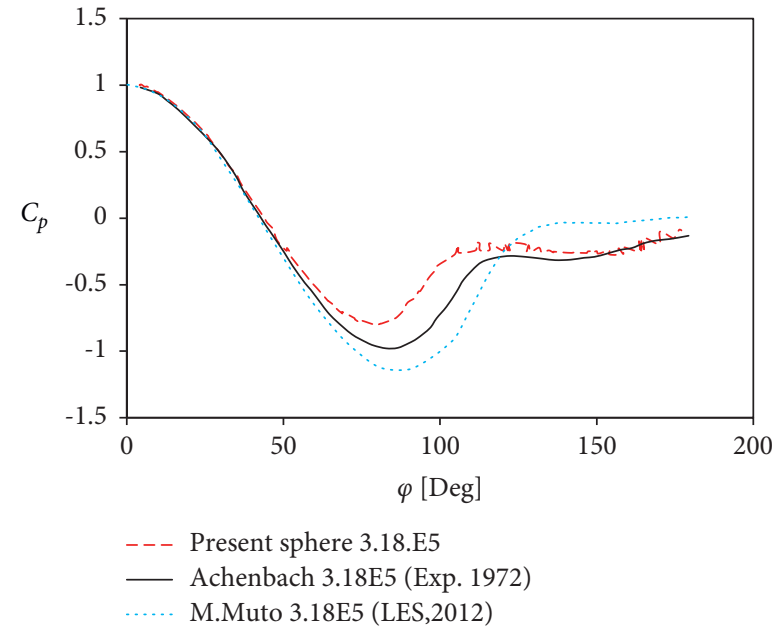

(b)

(a)

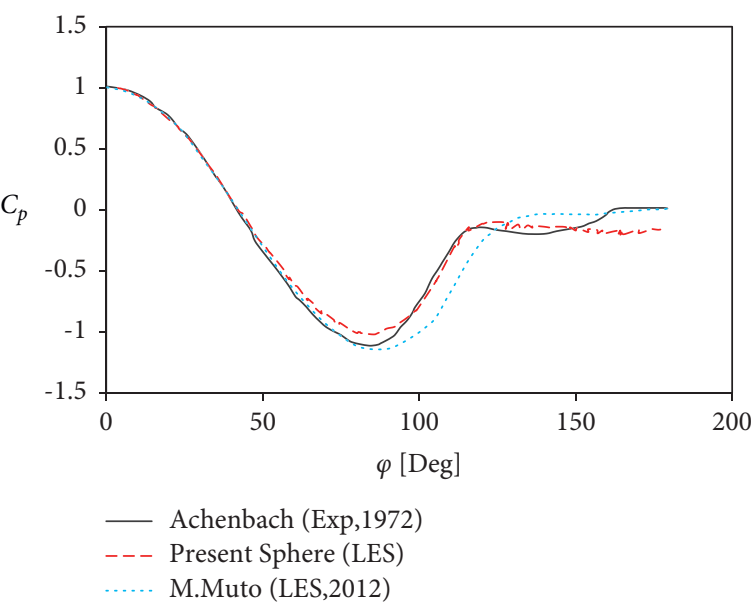

(c)

Figure 5: Pressure distributions on the surface of sphere (a) $\operatorname{Re}=1 \times 10^{4}$, (b) $\operatorname{Re}=3.18 \times 10^{5}$, and (c) $\operatorname{Re}=1.41 \times 10^{6}$.

TABLE 1: Calculated vs experimental drag coefficient.

\begin{tabular}{|c|c|c|c|}
\hline \multirow[b]{2}{*}{$\begin{array}{l}\text { Velocity } \\
(\mathrm{m} / \mathrm{s})\end{array}$} & \multicolumn{2}{|c|}{$C_{\mathrm{D}}$ experimental } & \multirow{2}{*}{$\begin{array}{c}C_{\mathrm{D}} \text { calculated } \\
\text { Random } \\
\text { orientation }\end{array}$} \\
\hline & $\begin{array}{c}0^{\circ} \\
\text { orientation }\end{array}$ & $\begin{array}{c}45^{\circ} \\
\text { orientation }\end{array}$ & \\
\hline 35 & 0.1937 & 0.202 & 0.15 \\
\hline
\end{tabular}

The following are the possible reasons for underprediction in drag coefficient values:

(i) Hong et al. [37] have confirmed through their wind tunnel experiments on ten different soccer balls in the supercritical Reynolds number range $\left(\mathrm{Re}=3.8 \times 10^{5}-5.0 \times 10^{5}\right)$ that the dimple-less soccer ball has a smaller drag value as compared to that of a soccer ball with surface dimples. The soccer ball used for the current numerical investigation has a smooth surface (dimple-less).

(ii) The offset between the calculated and experimental results can also be because of the difference in the soccer ball's orientation during wind tunnel and numerical experiments. During wind tunnel experiments, the soccer ball was fixed in two directions $\left(0^{\circ}\right.$ and $\left.45^{\circ}\right)$ [36]. However, a random orientation (different from the two directions considered in the experimental study) is selected in the present case.

(iii) The experimental drag coefficients also include the effects of flow interaction between the sphere and its supporting device, which could not be considered in the simulations [22].

Instantaneous and time-averaged contours of axial velocity around the sphere and soccer ball surfaces are shown in Figure 6. An early separation and a wider wake can be seen in the case of the sphere. However, the separation point moves toward a more downstream position in the soccer ball case and results in a narrow turbulent wake behind the soccer ball. These observations are consistent with previous literature [37-39]. In the case of the sphere, the boundary layer separation occurs at an angle of $105^{\circ}$ that is close to the $114 \pm 2^{\circ}$ measured by Achenbach [34]. However, in the case of a soccer ball, the separation was predicted at an angle of $110^{\circ}$. 


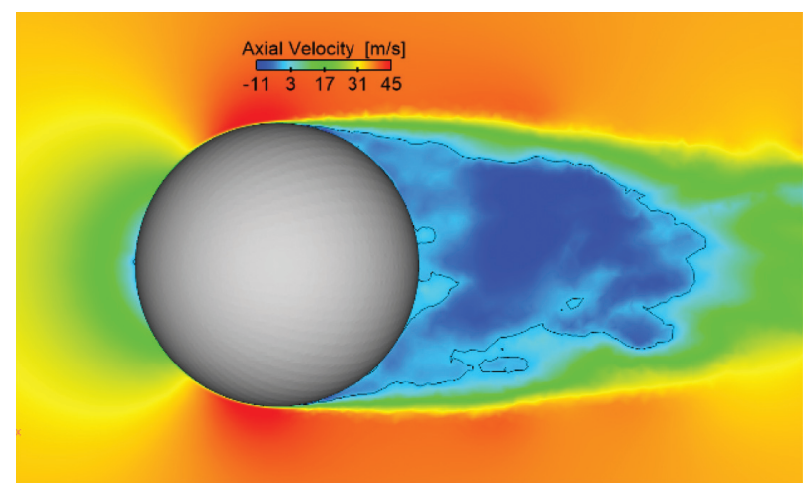

(a)

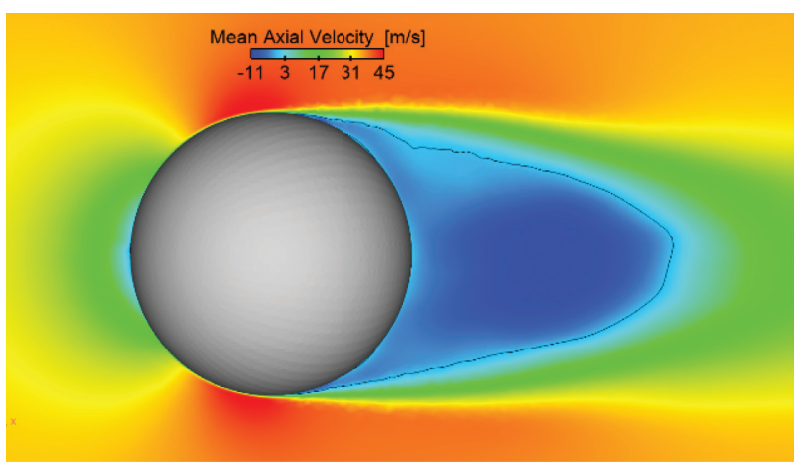

(c)

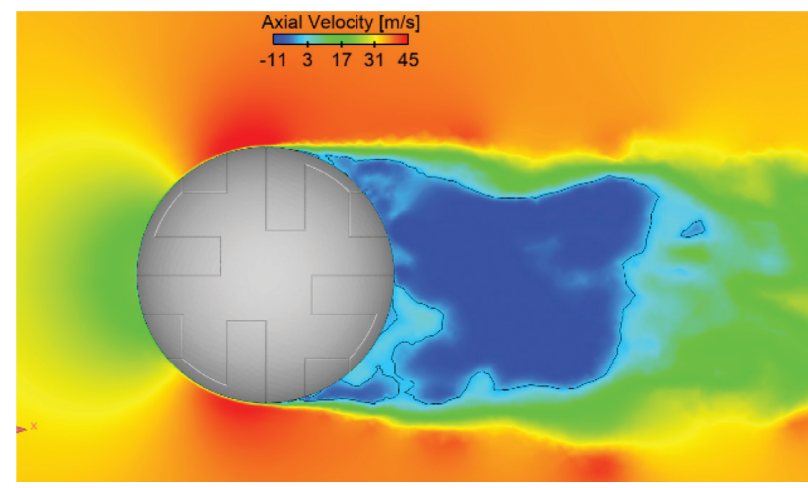

(b)

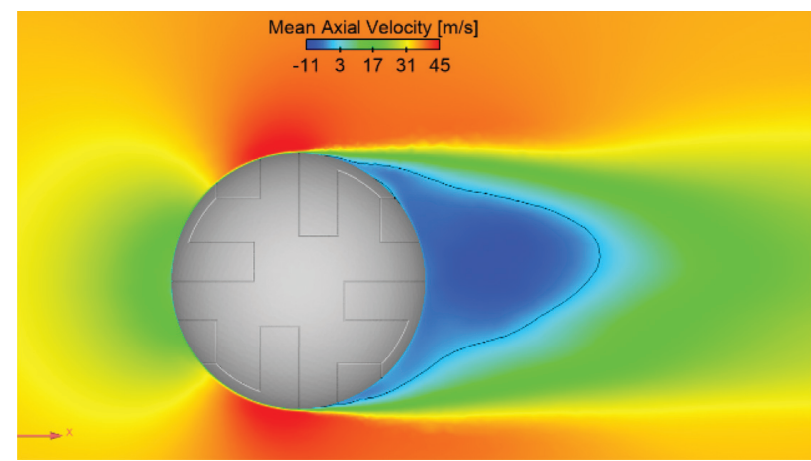

(d)

Figure 6: Instantaneous (top) and time average (bottom) contours of axial velocity around (a, c) the sphere and (b, d) the soccer ball. Solid lines represent zero axial velocities.

Similarly, the negative axial velocity region behind the sphere and soccer ball indicates a recirculation zone because of boundary layer detachment. The points where the streamwise velocity of the prism layer cell on the sphere/ soccer ball becomes negative are identified as boundary layer separation points. It can also be observed from Figures 6(c) and $6(\mathrm{~d})$ that in the case of the soccer ball, the size of the recirculation region is smaller, and the wake recovers more quickly as compared to the sphere case, confirming the evidence of the small drag coefficient in case of the soccer ball. The exact size of the recirculation region can be calculated from the mean streamwise velocity profile along the central line (presented in Figure 7). The recirculation region in the wake is where the mean velocity profiles drop below the zero value. Hence, the length of the recirculation region is the distance between the rear surface of the sphere/soccer ball and the point where the mean velocity restores to zero value. The time-averaged nondimensional recirculation length is 0.92 in the case of the sphere and 0.62 in the case of the soccer ball.

To get a further insight into near and far wake region characteristics of the smooth sphere and soccer ball, the time-averaged streamwise velocity distributions at different axial distances from the center of the smooth sphere and soccer ball are plotted in Figures 8(a)-8(f). The dips in these graphs indicate the positions in the wake, where the maximum fluctuations because of the vortex motions or coherent structures occur. Similarly, the points on the horizontal axis $(y / D)$ where the velocity is approximately

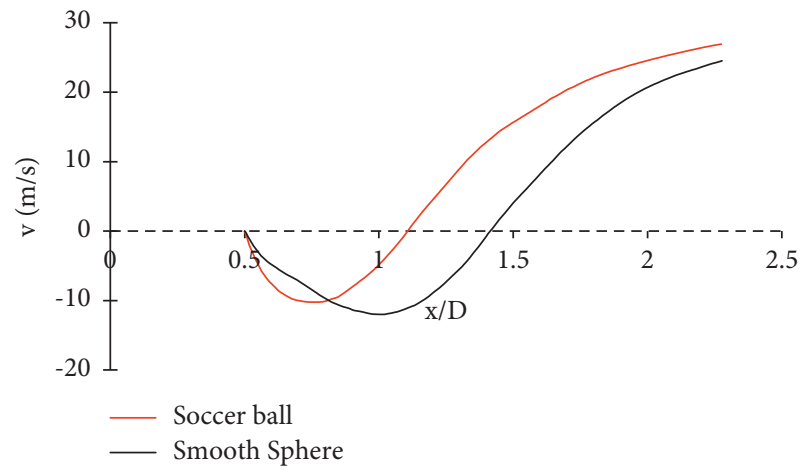

FIGURE 7: Time-averaged streamwise velocity distributions at $y=0$.

99\% of the free stream velocity correspond to the wake edges [40]. In the near wake $(0.5 \leq x / D \leq 1.2)$, the negative values of velocity indicate the existence of a mean recirculating region.

It is clear in Figures $8(\mathrm{a})-8(\mathrm{f})$ that the wake region behind the soccer ball is narrow and disappears faster than that behind the sphere. The small vortices generated because of the presence of the seam on the soccer ball surface draw more energy from the main flow and add it to the turbulent boundary layer. It improves the overall ability of the flow to remain attached to the surface and results in a significantly reduced wake produced behind the soccer ball.

Figures 9(a) and 9(b) provide the visualization of instantaneous vorticity near the sphere and soccer ball surface, respectively. The shear layer developed after flow separation 

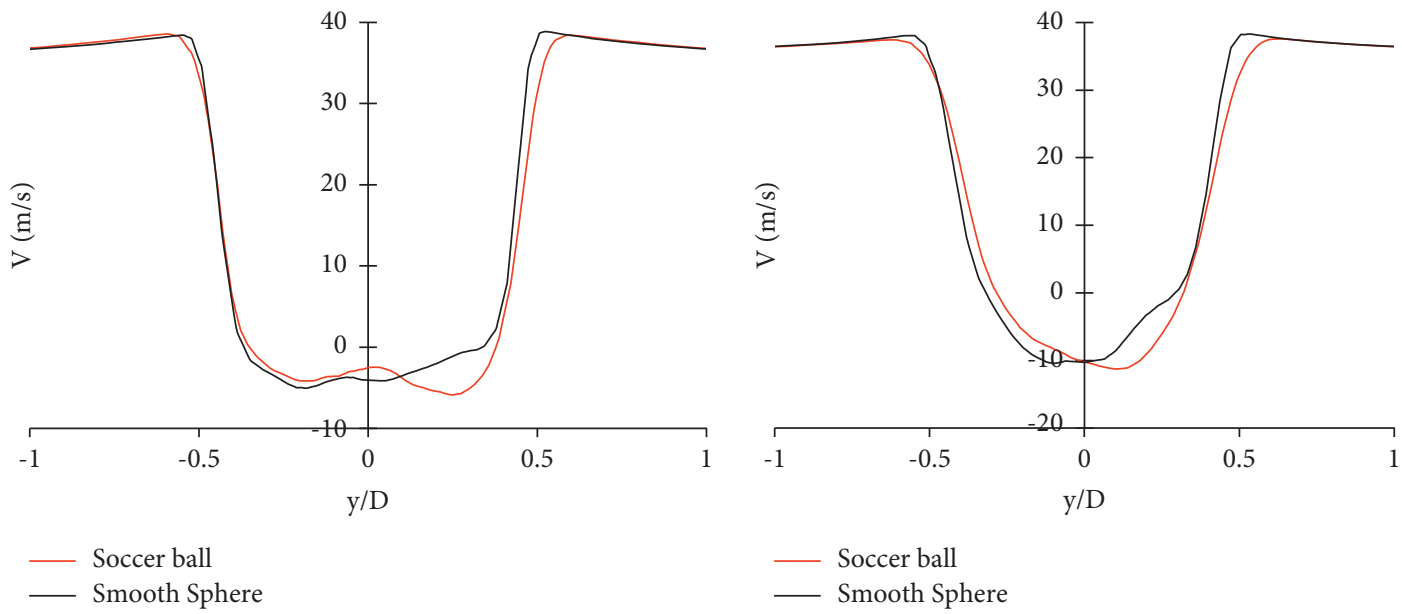

(a)
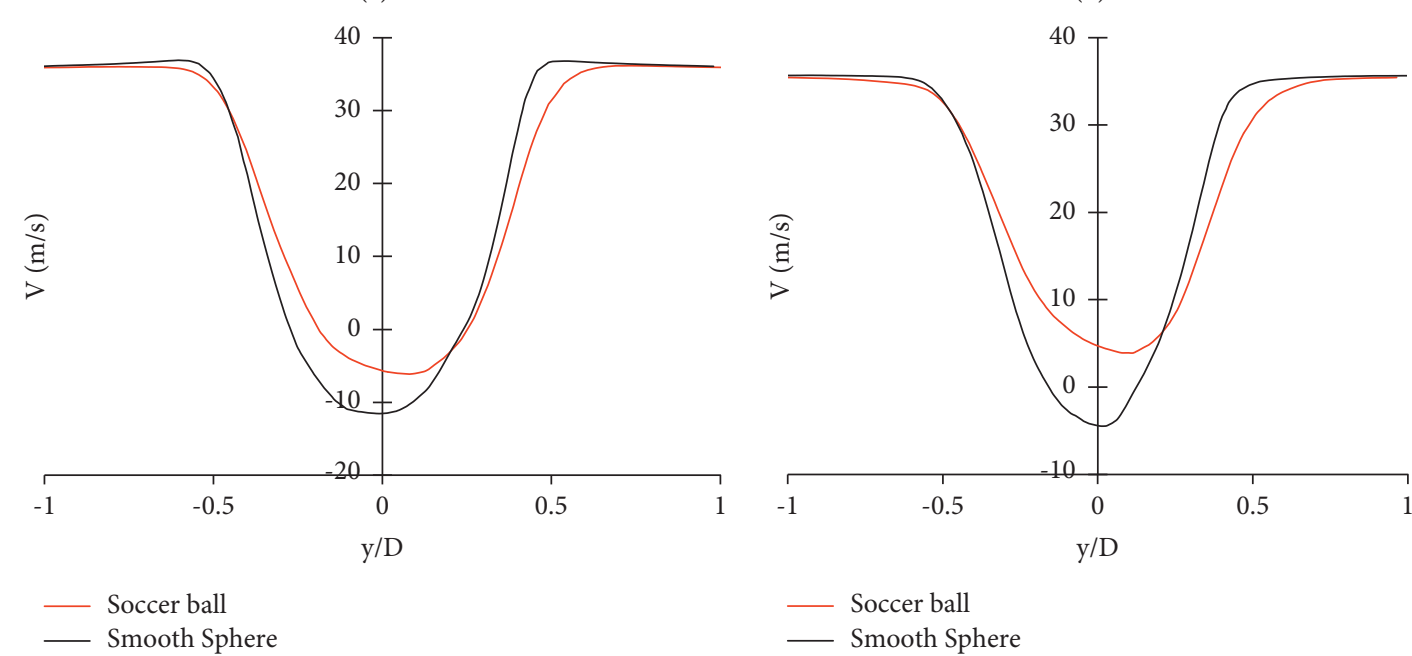

(c)
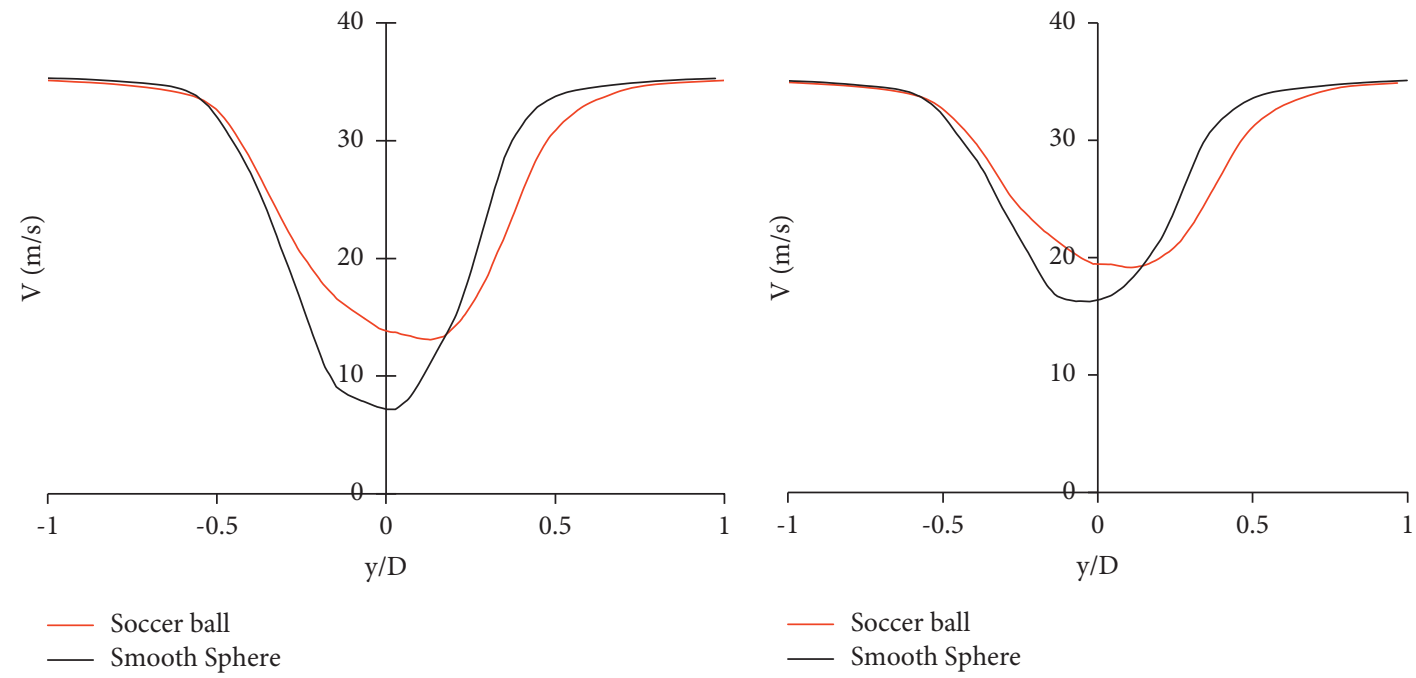

(e)

(f)

Figure 8: Variation of time-averaged streamwise velocity distributions at different axial distances. (a) $x / D=0.5$. (b) $x / D=0.75$. (c) $x / D=1$. (d) $x / D=1.2$. (e) $x / D=1.4$. (f) $x / D=1.65$. 


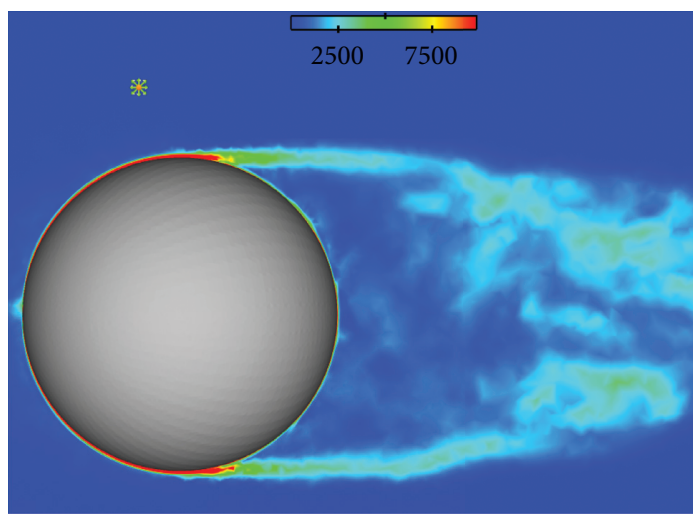

(a)

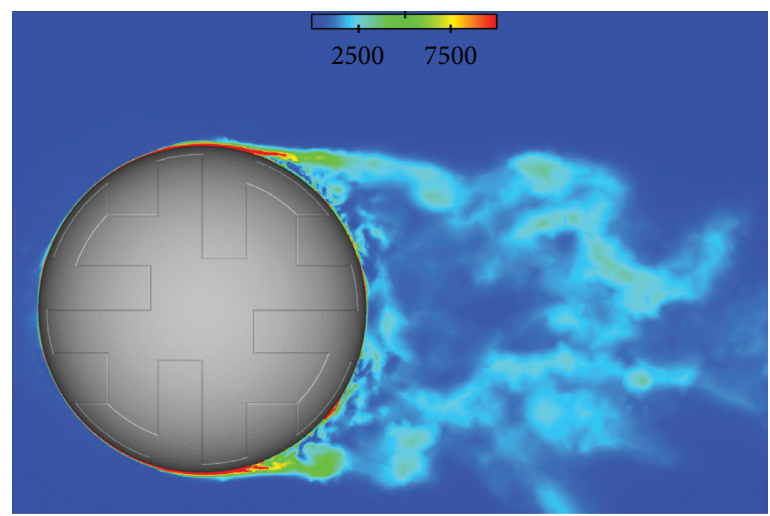

(b)

FIGURE 9: Contours of instantaneous vorticity near the surface of (a) sphere and (b) soccer ball.

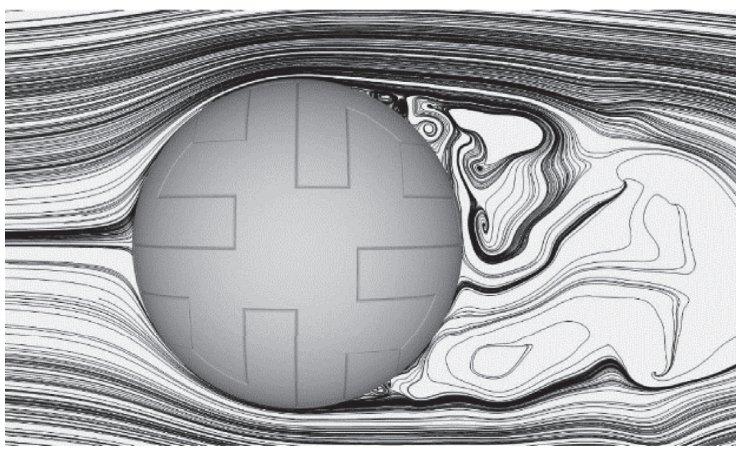

(a)

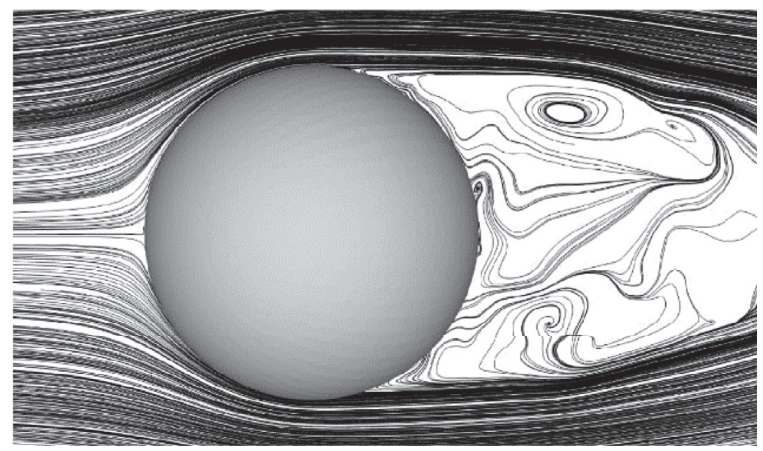

(b)

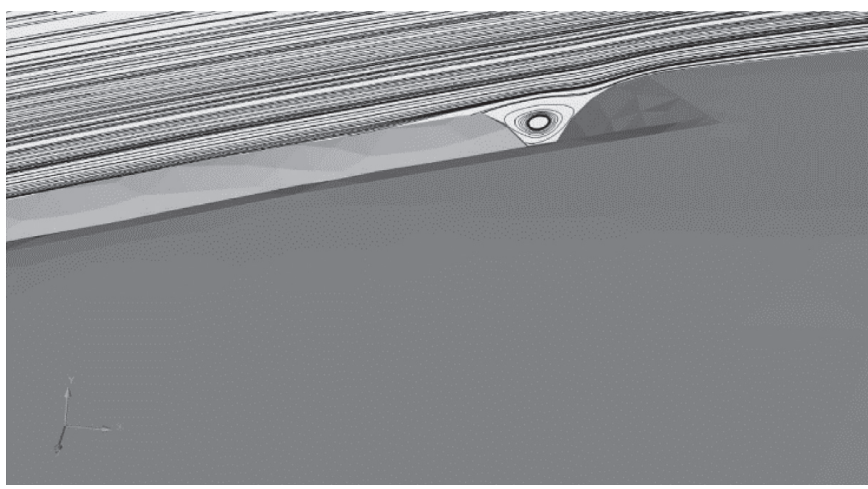

(c)

Figure 10: Flow streamlines around the (a) sphere and (b) soccer ball and (c) the zoomed-in view of the flow field inside the seam area.

from the sphere's surface appears to have slight instability until it travels some distance downstream from the separation point. On the other hand, in soccer balls, the flow becomes unstable after the boundary layer detachment and roll-up of the shear layer occur.

The effect of the existence of the seam on the soccer ball surface on the wake vortex is readily apparent in the side view of the flow streamlines around the sphere and soccer ball (shown in Figure 10). It can also be observed that several small-scale vortices are generated from the top and bottom regions of the soccer ball. On the other hand, large vortices can be seen in the wake region behind the sphere. To understand the reason for the difference in the vortex structure behind the soccer ball, a close-up view of the flow field is considered (Figure 10(c)). It can be observed that small eddies are generated in the seam area of the soccer ball. These small eddies interact with each other as they move downstream and form a complex flow.

In contrast, there is no eddy generation on the surface of the smooth sphere, and a large vortex is created in the wake region after the flow separates from the surface. Similar behavior was reported by Hong et al. [37] while experimentally measuring the aerodynamic forces of 11 different soccer balls with varied panel patterns. During their study, 


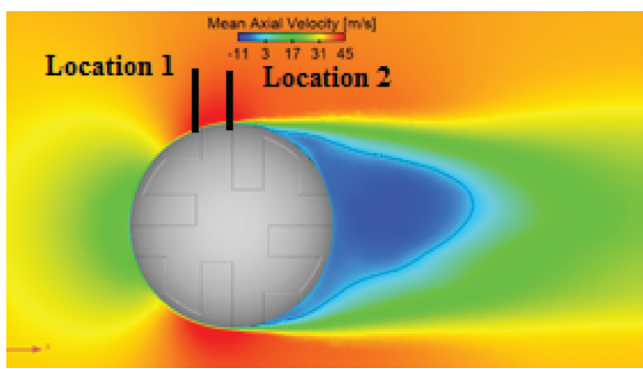

(a)

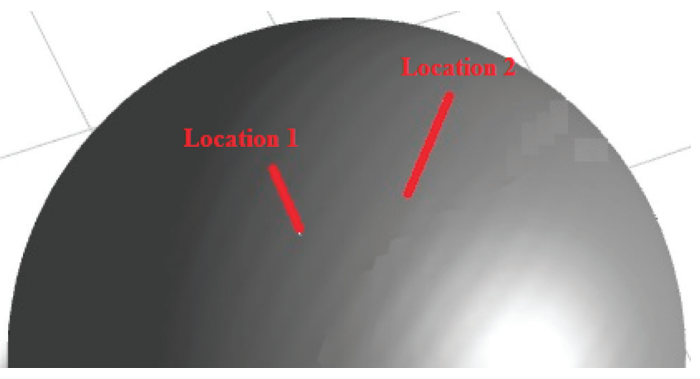

(c)

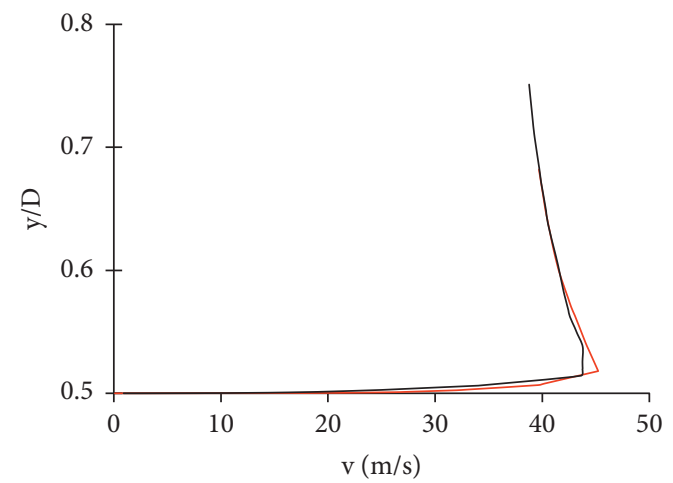

Soccer ball Smooth Sphere

(e)

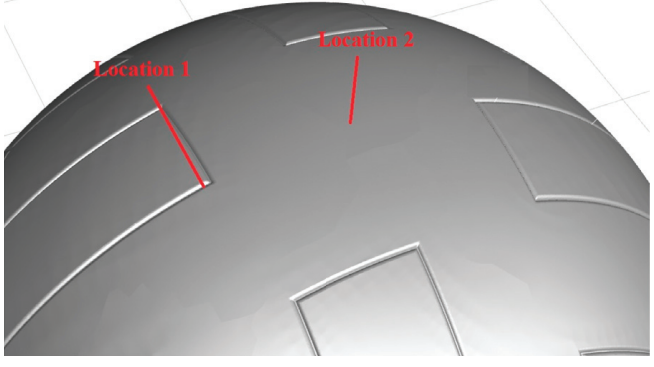

(b)

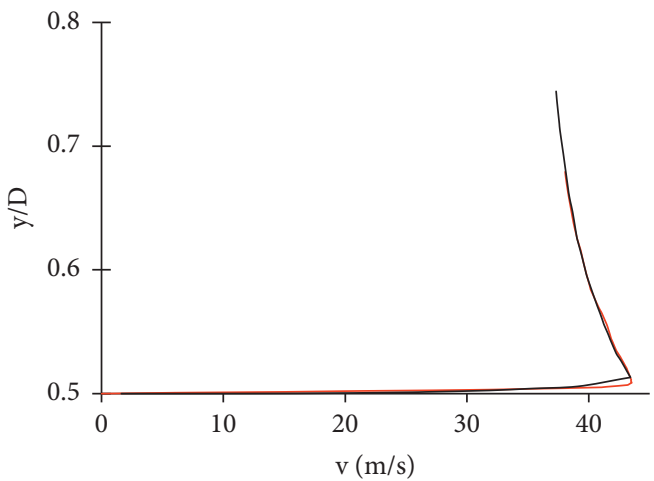

- Soccer ball

- Smooth Sphere

(d)

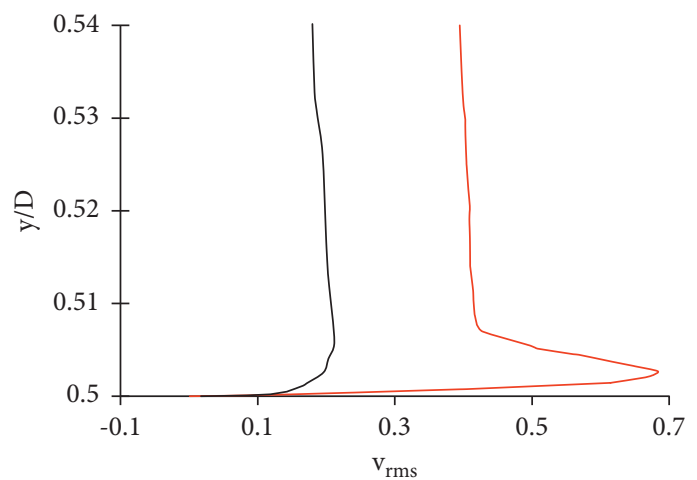

Soccer ball

— Smooth Sphere

(f)

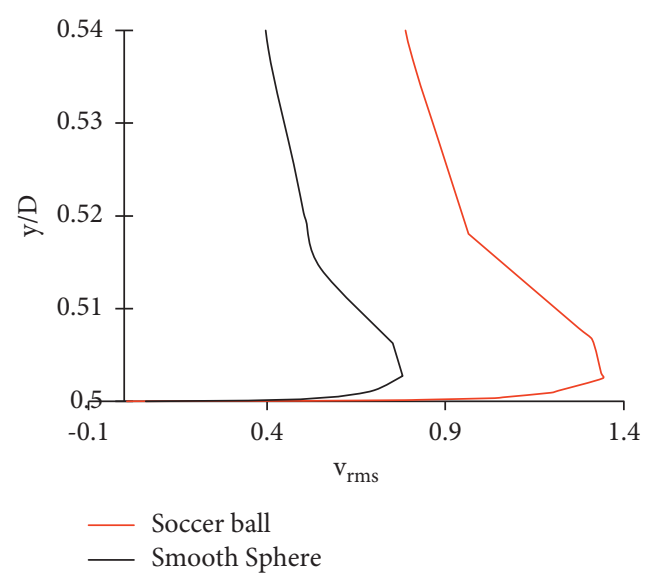

(g)

FiguRE 11: $(\mathrm{a}-\mathrm{c})$ Location of the measurement points on the soccer ball and smooth sphere surfaces, $(\mathrm{d}, \mathrm{f})$ time-averaged streamwise velocity and root mean square (RMS) streamwise velocity fluctuations at location 1 , and (e, g) time-averaged streamwise velocity and root mean square (RMS) streamwise velocity fluctuations at location 2 . 


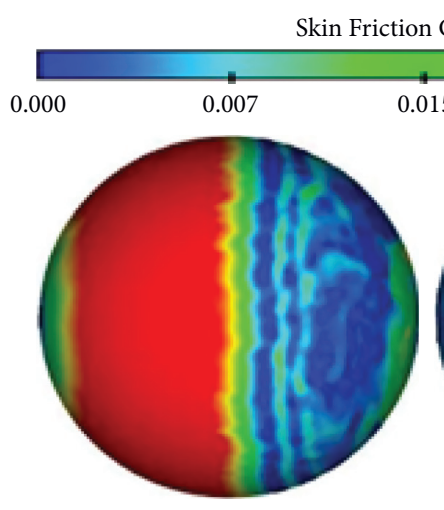

(a)

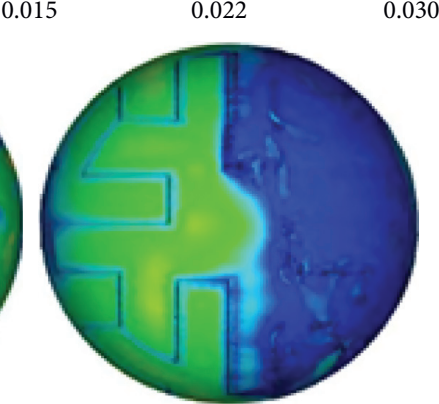

(b)

FIGURE 12: Skin friction coefficient contours on (a) smooth sphere and (b) soccer ball.

separation bubbles were found in each panel groove (seam) that acts as a triggering source for laminar to turbulent flow transition.

Figures 11(a)-11(g) provide more detailed insight into the effect of seams on the flow field, especially the boundary layer flow. Near-wall variations in the time-averaged streamwise velocity and root mean square (RMS) streamwise velocity fluctuations are captured at the two specific locations (locations $1 \& 2$ ) before the flow separation on the smooth sphere and soccer ball surfaces. Figures 11(f) and $11(\mathrm{~g})$ show the RMS streamwise velocity fluctuations profile as a function of normalized wall-normal distance at a point inside the seam area (at locations I) and a downstream point (location 2), respectively. In Figure 11(f), the significant increase in the velocity fluctuations confirms the presence of the vortex motion in the seam area on the soccer ball surface. The effect of the presence of seam on the boundary layer flow field is also felt at the downstream point (Location 2) depicted by the pronounced difference in the velocity fluctuation in the case of soccer ball (Figure 11(g)).

The skin friction coefficient distributions on the soccer balls' surface also show the effect of seam and panels. It can be observed from Figure 12 that the skin friction coefficient changes locally in regions near the seams because of localized small flow structures. Figure 13 shows the side view of the time average pressure distribution on the soccer ball and sphere surfaces. The high-pressure region near the central plan of the ball is because of significant flow separation in this area.

A more detailed understanding of the effect of the seam on the local flow behavior is provided by Figures 14 and 15 .

A comparison of the pressure coefficient distribution around the smooth sphere and soccer ball from the forward to the rear stagnation point is shown in Figure 14. Two distinct drops in the pressure coefficient plot are because of the generation of small-scale vortices inside the seam present at these points. A similar trend was reported by Hong et al. [19] while simulating the flow around a nonrotating twodimensional demonstration of the soccer ball. The author observed a spike in the skin friction coefficient and a distinct drop in the pressure coefficient at the seam location.

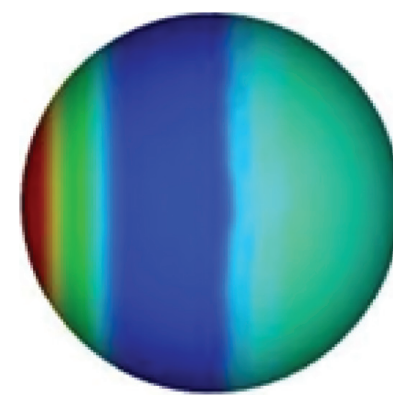

(a)

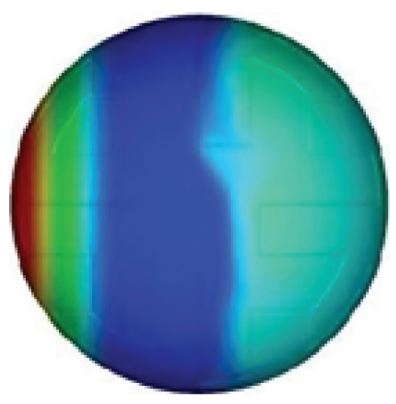

(b)

Mean Pressure $[\mathrm{Pa}]$

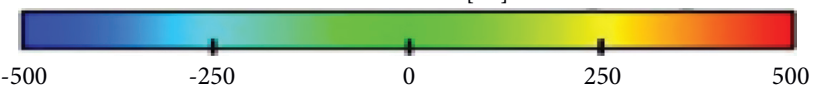

FIGURE 13: Mean pressure distribution on (a) smooth sphere and (b) soccer ball.

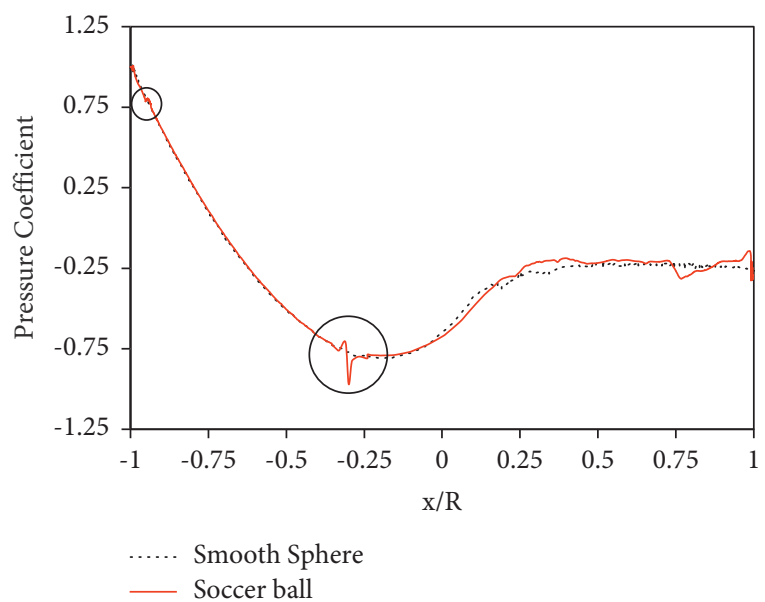

Figure 14: Comparison of the pressure coefficient distribution around the smooth sphere and soccer ball.

A more detailed insight into the effect of seams on separation is provided by the flow of the streamlines shown in Figure 15. Contrary to the smooth sphere, the separation point along the soccer ball varies more along the azimuthal direction. The variation in the location separation is because 


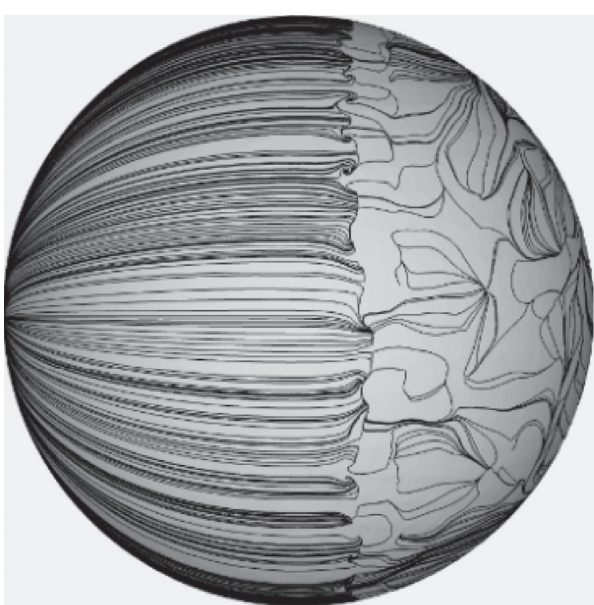

(a)

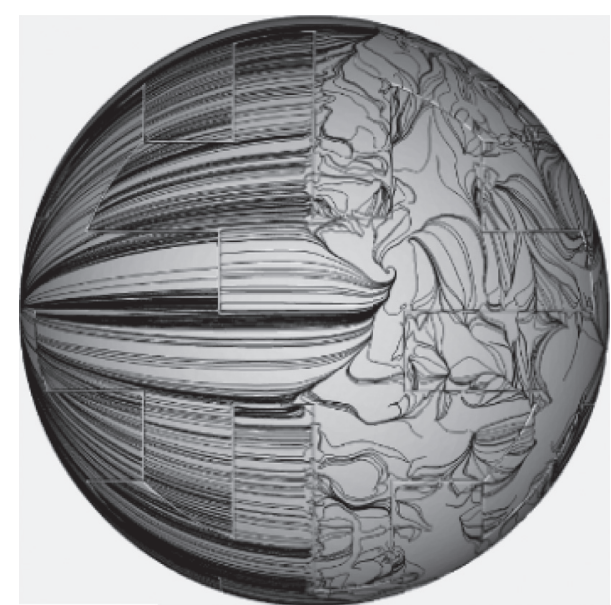

(b)

Figure 15: Comparison of streamlines of flow over (a) sphere and (b) soccer ball.

of the position of each seam and the mutual effect of multiple seamlines. These trends also matched the numerical and wind tunnel results of the different soccer balls performed by Barber et al. [22] and Hong et al. [35, 41], respectively.

\section{Conclusions}

The soccer balls with varied panel shape, number, and seam configurations have different aerodynamic behaviors and flight characteristics. This research aimed to simulate the flow around the modern soccer balls using high-fidelity large-eddy simulation techniques. The effect of panels and seam on the boundary layer flow separation and overall turbulent flow structure around the soccer ball are visualized. It is concluded that the delayed separation point and a relatively narrow wake have been observed in the soccer ball case. Small eddies are generated in the seam area of the soccer ball. These small eddies interact with each other as they move downstream and form a complex flow. The number of seams and their position on the soccer ball surface effects the location of the separation points. In the future, the numerical approach employed in current research will simulate the flow around the soccer ball with surface dimples and assess the effect of panel orientation on the aerodynamics of the static and spinning soccer balls.

\section{Data Availability}

All data are available from the corresponding author upon request.

\section{Conflicts of Interest}

The authors have no conflicts of interest, financial or otherwise.

\section{References}

[1] N. Gupta, N. Bhardwaj, G. M. Khan, and V. Dave, "Global trends of computational fluid dynamics to resolve real world problems in the contemporary era," vol. 6, no. 3, pp. 136-155, Current Biochemical Engineering, 2020.

[2] R. K. Hanna, "CFD in sport - a retrospective; 1992 - 2012," Procedia Engineering, vol. 34, pp. 622-627, 2012.

[3] E. Hala, "Wind performance for a stadium geometry," Research on Engineering Structures and Materials, vol. 6, no. 4, pp. 411-423, 2020.

[4] G. Losi, A. Bonzanini, A. Aquino, and P. Poesio, "Analysis of thermal comfort in a football stadium designed for hot and humid climates by CFD," Journal of Building Engineering, vol. 33, Article ID 101599, 2021.

[5] J. E. Goff, "A review of recent research into aerodynamics of sport projectiles," Sports Engineering, vol. 16, no. 3, pp. 137-154, 2013.

[6] M. J. Carré, S. R. Goodwill, and S. J. Haake, "Understanding the effect of seams on the aerodynamics of an association football," Proceedings of the Institution of Mechanical Engineers - Part C: Journal of Mechanical Engineering Science, vol. 219, no. 7, pp. 657-666, 2005.

[7] T. Asai, K. Seo, O. Kobayashi, and R. Sakashita, "Flow visualization on a real flight non-spinning and spinning soccer ball," The Engineering of Sport 6Developments for Sports, Springer-Verlag, vol. 1, pp. 327-332, , New York, USA, 2006.

[8] T. Asai, K. Seo, O. Kobayashi, and R. Sakashita, "Fundamental aerodynamics of the soccer ball," Sports Engineering, vol. 10, no. 2, pp. 101-109, 2007.

[9] L. Oggiano and L. Sætran, "Aerodynamics of modern soccer balls,” Procedia Engineering, vol. 2, no. 2, pp. 2473-2479, 2010.

[10] M. Passmore, D. Rogers, S. Tuplin, A. Harland, T. Lucas, and C. Holmes, "The aerodynamic performance of a range of FIFA-approved footballs," Proceedings of the Institution of Mechanical Engineers - Part P: Journal of Sports Engineering and Technology, vol. 226, no. 1, pp. 61-70, 2012. 
[11] T. Asai, S. Ito, K. Seo, and S. Koike, "Characteristics of modern soccer balls," Procedia Engineering, vol. 34, pp. 122-127, 2012.

[12] T. Asai and K. Seo, "Aerodynamic drag of modern soccer balls," SpringerPlus, vol. 2, no. 1, p. 171, 2013.

[13] F. Alam, H. Chowdhury, H. Moria, and F. K. Fuss, "A comparative study of football aerodynamics," Procedia Engineering, vol. 2, no. 2, pp. 2443-2448, 2010.

[14] F. Alam, H. Chowdhury, H. Chowdhury, H. Moria et al., Aerodynamics of contemporary FIFA soccer balls," Procedia Engineering, vol. 13, pp. 188-193, 2011.

[15] F. Alam, H. Chowdhury, S. George, I. Mustary, and G. Zimmer, "Aerodynamic drag measurements of FIFA-approved footballs," Procedia Engineering, vol. 72, pp. 703-708, 2014.

[16] F. Alam, H. Chowdhury, B. Loganathan, I. Mustary, and S. Watkins, "Aerodynamic drag of contemporary soccer balls," in Proceedings of the 19th Australasian Proceedings of Fluid Mechanics Conference, Melbourne, Australia, December, 2014.

[17] S. Hong, K. Sakamoto, Y. Washida, M. Nakayama, and T. Asai, "The influence of panel orientation on the aerodynamics of soccer balls," Procedia Engineering, vol. 72, pp. 786-791, 2014.

[18] J. E. Goff, T. Asai, and S. Hong, "A comparison of Jabulani and Brazuca non-spin aerodynamics," Proceedings of the Institution of Mechanical Engineers - Part P: Journal of Sports Engineering and Technology, vol. 228, no. 3, pp. 188-194, 2014.

[19] S. Hong, J. E. Goff, and T. Asai, "Effect of a soccer ball's surface texture on its aerodynamics and trajectory," Proceedings of the Institution of Mechanical Engineers - Part P: Journal of Sports Engineering and Technology, vol. 233, no. 1, pp. 67-74, 2019.

[20] S. Hong and T. Asai, "Aerodynamic effects of dimples on soccer ball surfaces," Heliyon, vol. 3, no. 10, Article ID e00432, 2017.

[21] S. Barber, S. J. Haake, and M. Carré, "Using CFD to understand the effects of seam geometry on soccer ball aerodynamics," in The Engineering of Sport 6, pp. 127-132, Springer, New York, USA, 2006.

[22] S. Barber, S. B. Chin, and M. J. Carré, "Sports ball aerodynamics: a numerical study of the erratic motion of soccer balls," Computers \& Fluids, vol. 38, no. 6, pp. 1091-1100, 2009.

[23] P. Jalilian, P. K. Kreun, M. M. Makhmalbaf, and W. W. Liou, "Computational aerodynamics of baseball, soccer ball and volleyball," American Journal of Sports Science, vol. 2, no. 5, pp. 115-121, 2014.

[24] A. Rohr, A Computational Fluid Dyndamics (CFD) Analysis of the Aerodynamic Effects of the Seams on a Two-Dimensional Representation of a Soccer Ball, California Polytechnic State University, San Luis Obispo, 2018.

[25] S. B. Hussain, S. I. A. Shah, and A. Shahzad, "Optimization and aerodynamic design of a soccer ball using numerical analysis," in Proceedings of the 2020 International Conference on Engineering and Emerging Technologies (ICEET), pp. 1-7, Lahore, Pakistan, Febrauary. 2020.

[26] T. Asai, Y. Nakanishi, N. Akiyama, and S. Hong, "Flow visualization of spinning and nonspinning soccer balls using computational fluid dynamics," Applied Sciences, vol. 10, no. 13 , p. $4543,2020$.

[27] H. K. Versteeg and W. Malalasekera, An Introduction to Computational Fluid Dynamics: The Finite Volume Methodpp. 9-38, Pearson Education, London, UK, 2nd ed. edition, 2007.

[28] D. G. Fox and D. K. Lilly, "Numerical simulation of turbulent flows," Reviews of Geophysics, vol. 10, no. 1, pp. 51-72, 1972.
[29] P. Moin and K. Mahesh, "Direct numerical simulation: a tool in turbulence research," Annual Review of Fluid Mechanics, vol. 30, no. 1, pp. 539-578, 1998.

[30] J. Ding, W. Yin, and Y. Ma, "Large eddy simulation and flow field analysis of car on the bridge under turbulent crosswind," Mathematical Problems in Engineering, vol. 2021, Article ID 7579696, 10 pages, 2021.

[31] Y. Zhiyin, "Large-eddy simulation: past, present and the future," Chinese Journal of Aeronautics, vol. 28, no. 1, pp. 11-24, 2015.

[32] ANSYS, Inc, LES-specific Solution Strategies, pp. 747-748, ANSYS Fluent Users Guide, Canonsburg, PA, USA, 2013.

[33] W. Sidebottom, A. Ooi, and D. Jones, "A parametric study of turbulent flow past a circular cylinder using large eddy simulation," Journal of Fluids Engineering, vol. 137, no. 9, 2015.

[34] E. Achenbach, "Experiments on the flow past spheres at very high Reynolds numbers," Journal of Fluid Mechanics, vol. 54, no. 3, pp. 565-575, 1972.

[35] M. Muto, M. Tsubokura, and N. Oshima, "Negative Magnus lift on a rotating sphere at around the critical Reynolds number," Physics of Fluids, vol. 24, no. 1, Article ID 014102, 2012.

[36] J. E. Goff, S. Hong, and T. Asai, "Aerodynamic and surface comparisons between telstar 18 and Brazuca," Proceedings of the Institution of Mechanical Engineers - Part P: Journal of Sports Engineering and Technology, vol. 232, no. 4, pp. 342$348,2018$.

[37] S. Hong, T. Asai, and K. Seo, "Visualization of air flow around soccer ball using a particle image velocimetry," Scientific Reports, vol. 5, no. 1, Article ID 15108, 2015.

[38] J. E. Goff, S. Hong, and T. Asai, "Effect of a soccer ball's seam geometry on its aerodynamics and trajectory," Proceedings of the Institution of Mechanical Engineers - Part P: Journal of Sports Engineering and Technology, vol. 234, no. 1, pp. 19-29, 2020.

[39] Y. Sakamoto, S. Ito, and M. Hiratsuka, "Difference of Reynolds crisis aspects on soccer balls and their panels," Proceedings, vol. 49, no. 1, p. 117, 2020.

[40] G. Fabris, "Higher-order statistics of turbulent fluctuations in the plane wake," Physics of Fluids, vol. 26, no. 6, pp. 1437-1445, 1983.

[41] S. Hong, T. Asai, and K. Seo, "Flow visualization around panel shapes of soccer ball," Procedia Engineering, vol. 112, pp. 391-394, 2015. 